\title{
An unexpected SCLC diagnosed by electromagnetic navigation bronchoscopy
}

\author{
Yuanyang Lai ${ }^{1}$, Dong Sun ${ }^{2}$, Peilong Bao ${ }^{1}$, Weimiao Li ${ }^{1}$, Xiaofei $\mathrm{Li}^{1}$, Jinbo Zhao ${ }^{1}$ \\ ${ }^{1}$ Department of Thoracic Surgery, ${ }^{2}$ Department of Cardiology, Tangdu Hospital, The Air Force Medical University, Xi'an 710038, China \\ Correspondence to: Xiaofei Li, MD; Jinbo Zhao, MD. Department of Thoracic Surgery, Tangdu Hospital, The Air Force Medical University, Xi'an \\ 710038, China. Email: lxfchest@fmmu.edu.cn; zhaojinbo@aliyun.com.
}

Submitted May 22, 2018. Accepted for publication Sep 25, 2018.

doi: $10.21037 /$ jtd.2018.10.20

View this article at: http://dx.doi.org/10.21037/jtd.2018.10.20

\section{Introduction}

Lung cancer remains the leading cause of cancer-related death in China and the United States $(1,2)$. Small cell lung carcinoma (SCLC) constitutes $10-15 \%$ of all lung cancer cases (3). Owing to its rapid volume-double time, high growth fraction and greater propensity for early hematogenous spread, it's considered the most aggressive form of lung cancer, its overall 5 -year survival rate is around $7 \%$. Traditionally, SCLCs present as a large hilar mass and bulky lymphadenopathy, whereas a peripheral SCLC is rather uncommon. One case with an unexpected peripheral SCLC was reported in our institute. After pathologically diagnosed as SCLC assisted by electromagnetic navigation bronchoscopy-guide trans-bronchial needle aspiration (ENB-TBNA), the patient underwent a standard videoassisted lobectomy and lymphadenectomy and recovered well. The confirmatory procedures as well as imaging demonstrations of peripheral SCLC will be discussed by reporting this case and reviewing related literature.

\section{Case presentation}

A 53-year-old female was admitted due to a nodular lesion in the right lower lobe. The patient was free from any respiratory, neurological, or endocrine paraneoplastic symptoms. She had no smoking or radioactive substances exposure history. No history of recurrent pulmonary infections including tuberculosis or family history of cancer was present.

Physical examinations and laboratory tests were unremarkable, particularly the tumor biomarkers including CEA, FRT, NSE, CA125, CYFRA21-1, CA50, SCC were negative.

Computed tomography with contrast of the chest (Figure 1) showed a $1.3 \mathrm{~cm} \times 1.4 \mathrm{~cm}$ well-defined solitary nodule adjacent to a segmentary artery in the right anterior basal segment. The lesion was homogenous in density; the CT value in the arterial phase was $30 \mathrm{HU}$ and that of the venous phase $48 \mathrm{HU}$, making the imaging diagnosis a bronchial mucous plug or a pulmonary cyst. Conventional bronchoscopy demonstrated a negative result.

The patient adopted one of the 3 recommendations and underwent an ENB-TBNA (others were 1-month followup and an immediate lobectomy). The ENB-TBNA in the case reported was performed by a bronchoscopist in our institute, SuperDimension Bronchus System (SDBS, Herzliya, Israel) was used for navigation, and TBNA was performed using a 22-gauge cytology needle. ENBTBNA successfully produced a sample (Figure 2) and hematoxylin-eosin staining and immunohistochemistry confirmed it to be a SCLC. A subsequent PET/CT scan (Figure 3) suggested that it was a limited stage disease without metastatic lymphadenopathy. A multidisciplinary team consultation, consisting of a pulmonologist, a thoracic surgeon, an oncologist and a radiologist was done, which determined the patient was at the cT1N0M0 and stage IA2. EBUS-TBNA was recommended to proceed pathological mediastinal staging according to NCCN guidelines; despite this, the patient refused further invasive diagnostic procedures and demanded surgical treatment. Considering PET/CT suggested no metastatic lymphadenopathy and that even N1 SCLC disease benefit from surgery (4,5), we believed straightforward surgery was reasonable and a video-assisted right lower lobe resection and systematic 


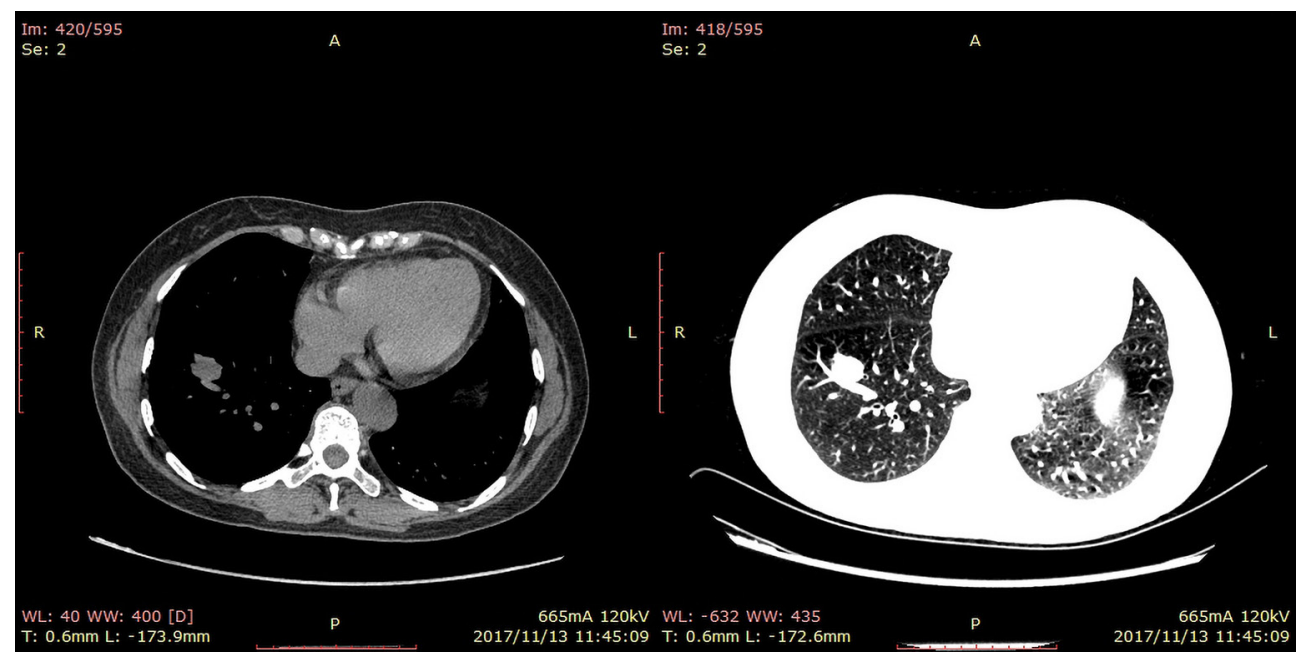

Figure 1 Computed tomography of the chest.

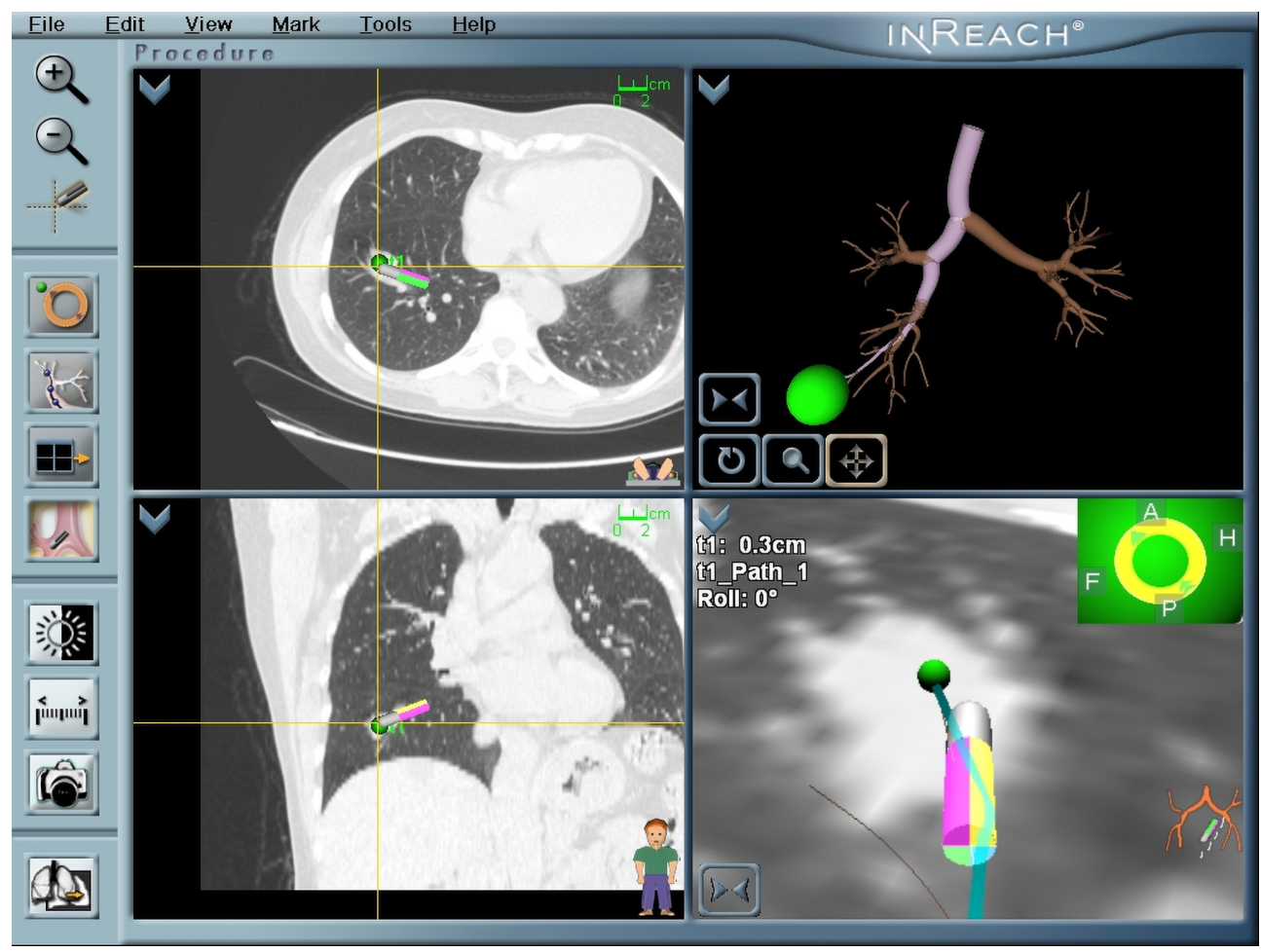

Figure 2 ENB-TBNA procedure. ENB-TBNA, electromagnetic navigation bronchoscopy-guide trans-bronchial needle aspiration.

lymphadenectomy of stations 2R, 3a, 4R, 7, 9R, 10R, 11R and $12 \mathrm{R}$ was performed under the patient's consent. The patient recovered well from the surgery with 5 -day chest drainage and 7-day hospital stay. Post-operation pathology confirmed it was a right lower lobe SCLC with stations 12R, 11R, 10R metastatic lymphadenopathies. Adjuvant chemotherapy and prophylactic cranial irradiation are to be followed.

\section{Discussion and conclusions}

SCLC typically manifests as a hilar mass and bulky 


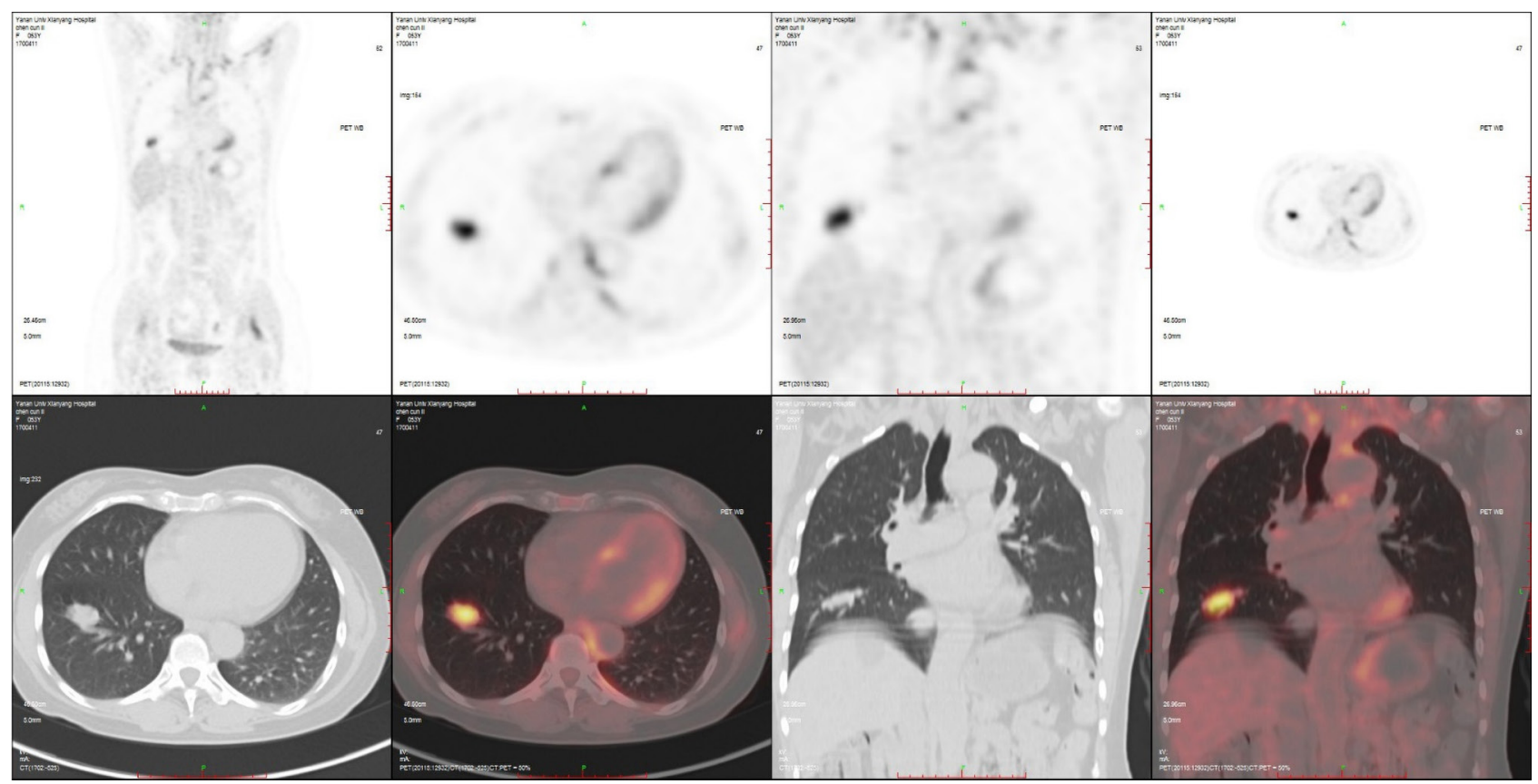

Figure 3 PET/CT scan findings. PET/CT, positron emission tomography/computed tomography.

mediastinal lymphadenopathy and symptoms of widespread metastatic diseases; it's long been believed to be the centraltype lung cancer, and the central-located SCLC accounted for about $60 \%$ of all the cases (6). Recently, occurrence of peripheral-located SCLC seems to be growing (7-9), which can partly be attributed to the advent of lung cancer screening with low-dose computed tomography (LDCT). Peripheral SCLCs seem to mean less hilar and mediastinum involvement, less extended stage cases, and less distant metastasis. Kanaji et al. (9) reported better survival rates in patients with peripheral-type SCLC, and concluded that it resulted from more frequent surgical treatment for peripheral located SCLC, better PS scores, less tumor burden and more limited stage diseased of such cases. Since surgery on high selected cT0-1N0-1 SCLC patients can yield a favorable 5-year survival rates of 40-60\% (5,10-13), early detection and surgical intervention in peripheral SCLC cases may be essential to improve the prognosis of the aggressive malignancy.

The treatment modality of peripheral cT0-1N0-1 SCLC is be lobectomy followed by adjuvant chemotherapy and prophylactic cranial irradiation (14); whereas the choice of confirmatory procedure of a peripheral lesion depends on available resources, cost performance, patient preference, practitioner expertise, etc. Currently, the most frequently- used procedures embrace conventional bronchoscopyguided transthoracic needle aspiration, CT-guided transthoracic needle aspiration, EBUS-guided transthoracic needle aspiration and more aggressive immediate surgery. Apart from the advantages, the drawbacks of the procedures mentioned above should be concerning. First, conventional bronchoscopy has a poor yield between $14 \%$ to $62 \%$ for small peripheral lesions (15-17). Second, while CT-guided core/fine needle aspiration does have higher sensitivity and precision for peripheral disease, its higher rates of complications such as pneumothorax and bleeding should not be overlooked (18-21). Third, previous studies (22-26) found that the diagnostic sensitivity of EBUS-TBNA ranged from $74.5 \%$ to $92 \%$ involved with sarcoidosis, lung cancer staging, or undiagnosed mediastinal lymphadenopathy, and the figure was similar with that of ENB-TNBA; whereas the figure dropped when focusing on diagnoses of lung nodules ( $46 \%$ to $88 \%$ ) (27-32), noticeably in some studies the high upper end resulted from the application of ROSE. This implies that ultrasound guiding techniques may be inferior to ENB on diagnosing lung nodules, especially peripheral lesions. Fourth, surgery is never a good option for the elderly patients or patients with higher Charlson Comorbidity Index, even though it can avoid pitfalls of less invasive procedures for low- to intermediate-risk patients 
with suspected cancer; and a lobectomy for small peripheral benign nodules may be much more aggressive.

Electromagnetic Navigation Bronchoscopy can be a good option under the circumstances similar to the case reported. It is recommended for detecting peripheral lesions that are hard to reach with conventional bronchoscopy (33), and its diagnostic yield varies from $60 \%$ to $87.5 \%$ (34-38), with a pooled sensitivity more than $71 \%$ and accuracy more than $73.9 \%(37,38)$. Moreover it is safer and the risk of pneumothorax is around $3.1 \%$ (37), whereas pneumothorax following transthoracic needle lung biopsy is $15 \%$ and hemorrhage $1 \%(19)$. Thus, ENB is an effective and safe procedure to investigate peripheral lung lesions; in the cases of peripheral SCLC, it can yield earlier diagnosis, lead to earlier interventions including surgery, and provide the chance for cure.

ENB's higher safety and yield come at a cost. Owing to its low cost-effectiveness ratio, ENB is not as prevalent as bronchoscopy-guided and CT-guided transthoracic needle aspiration. In most cases, computed tomography still plays an important role in the diagnosis of SCLC. Efforts were made to summarize recognizable CT features of SCLC (39-42). It seems that peripheral SCLC is a lobulated $(69.6 \%)$ or a well-defined $(66.1 \%, 37 / 56)$ mass rather than a spiculated mass (17.9\%, 10/56). Notably, Yabuuchi et al. (39) reported that all the 12 peripheral lesions were homogenous in density. These findings can provide some references for imaging diagnosis, even though they may be nonspecific. On the other hand, as positron emission tomography (PET) with $\left[{ }^{18} \mathrm{~F}\right]$ fluorodeoxy-D-glucose (FDG) emerges as an accurate and superior tool for NSCLC staging, studies aimed at exploring the similar utility of PET in SCLC. A prospective study of 120 consecutive patients with newly detected SCLC concluded that complete agreement between FDG-PET results and other staging procedures were observed in 75 patients (43), but there seemed to be no pathological confirmations. The divergence between PET and pathology does exist, as depicted in the case reported above, metastatic lymphadenopathy in SCLC may not be detected by PET/CT scan. We speculate that the underlying reason is lower tumor burden in metastatic lymph nodes in early stage peripheral SCLC. Thus, the precise workup of peripheral SCLC should be comprehensive, and invasive procedures could be recruited if necessary.

All in all, we conclude that Electromagnetic Navigation Bronchoscopy might be useful for peripheral nodes diagnosis and avoidance of futile surgeries. Particularly in the case reported, Electromagnetic Navigation Bronchoscopy can be beneficial to diagnose peripheral SCLC at the early stage, leading to earlier surgical intervention and providing the chance for cure.

\section{Acknowledgements}

None.

\section{Footnote}

Conflicts of Interest: The authors have no conflicts of interest to declare.

Informed Consent: Written informed consent was obtained from the patient for publication of this manuscript and any accompanying images.

\section{References}

1. Chen $\mathrm{W}$, Zheng R, Baade PD, et al. Cancer statistics in China, 2015. CA Cancer J Clin 2016;66:115-32.

2. Siegel RL, Miller KD, Jemal A. Cancer statistics, 2018. CA Cancer J Clin 2018;68:7-30.

3. Riaz SP, Luchtenborg M, Coupland VH, et al. Trends in incidence of small cell lung cancer and all lung cancer. Lung Cancer 2012;75:280-4.

4. Stahel R, Thatcher N, Fruh M, et al. 1st ESMO Consensus Conference in lung cancer; Lugano 2010: small-cell lung cancer. Ann Oncol 2011;22:1973-80.

5. Yokouchi H, Ishida T, Yamazaki S, et al. Prognostic impact of clinical variables on surgically resected smallcell lung cancer: Results of a retrospective multicenter analysis (FIGHT002A and HOT1301A). Lung Cancer 2015;90:548-53.

6. Bandoh S, Fujita J, Ueda Y, et al. Expression of carcinoembryonic antigen in peripheral- or central-located small cell lung cancer: its clinical significance. Jpn J Clin Oncol 2001;31:305-10.

7. Miyauchi E, Motoi N, Ono H, et al. Distinct Characteristics of Small Cell Lung Cancer Correlate With Central or Peripheral Origin: Subtyping Based on Location and Expression of Transcription Factor TTF-1. Medicine (Baltimore) 2015;94:e2324.

8. Nobashi T, Koyasu S, Nakamoto Y, et al. Prognostic value of fluorine-18 fludeoxyglucose positron emission tomography parameters differs according to primary tumour location in small-cell lung cancer. Br J Radiol 
2016;89:20150618.

9. Kanaji N, Sakai K, Ueda Y, et al. Peripheral-type small cell lung cancer is associated with better survival and higher frequency of interstitial lung disease. Lung Cancer 2017;108:126-33.

10. Inoue M, Miyoshi S, Yasumitsu T, et al. Surgical results for small cell lung cancer based on the new TNM staging system. Thoracic Surgery Study Group of Osaka University, Osaka, Japan. Ann Thorac Surg 2000;70:1615-9.

11. Brock MV, Hooker CM, Syphard JE, et al. Surgical resection of limited disease small cell lung cancer in the new era of platinum chemotherapy: Its time has come. J Thorac Cardiovasc Surg 2005;129:64-72.

12. Lim E, Belcher E, Yap YK, et al. The role of surgery in the treatment of limited disease small cell lung cancer: time to reevaluate. J Thorac Oncol 2008;3:1267-71.

13. Shields TW, Higgins GJ, Matthews MJ, et al. Surgical resection in the management of small cell carcinoma of the lung. J Thorac Cardiovasc Surg 1982;84:481-8.

14. Combs SE, Hancock JG, Boffa DJ, et al. Bolstering the case for lobectomy in stages I, II, and IIIA small-cell lung cancer using the National Cancer Data Base. J Thorac Oncol 2015;10:316-23.

15. Wallace JM, Deutsch AL. Flexible fiberoptic bronchoscopy and percutaneous needle lung aspiration for evaluating the solitary pulmonary nodule. Chest 1982;81:665-71.

16. Weiser TS, Hyman K, Yun J, et al. Electromagnetic navigational bronchoscopy: a surgeon's perspective. Ann Thorac Surg 2008;85:S797-801.

17. Baaklini WA, Reinoso MA, Gorin AB, et al. Diagnostic yield of fiberoptic bronchoscopy in evaluating solitary pulmonary nodules. Chest 2000;117:1049-54.

18. Wiener RS, Schwartz LM, Woloshin S, et al. Populationbased risk for complications after transthoracic needle lung biopsy of a pulmonary nodule: an analysis of discharge records. Ann Intern Med 2011;155:137-44.

19. Sachdeva M, Ronaghi R, Mills PK, et al. Complications and Yield of Computed Tomography-Guided Transthoracic Core Needle Biopsy of Lung Nodules at a High-Volume Academic Center in an Endemic Coccidioidomycosis Area. Lung 2016;194:379-85.

20. Heerink WJ, de Bock GH, de Jonge GJ, et al. Complication rates of CT-guided transthoracic lung biopsy: meta-analysis. Eur Radiol 2017;27:138-48.

21. Hiraki T, Mimura H, Gobara H, et al. Incidence of and risk factors for pneumothorax and chest tube placement after CT fluoroscopy-guided percutaneous lung biopsy: retrospective analysis of the procedures conducted over a 9-year period. AJR Am J Roentgenol 2010;194:809-14.

22. Agarwal R, Srinivasan A, Aggarwal AN, et al. Efficacy and safety of convex probe EBUS-TBNA in sarcoidosis: a systematic review and meta-analysis. Respir Med 2012;106:883-92.

23. Gupta D, Dadhwal DS, Agarwal R, et al. Endobronchial ultrasound-guided transbronchial needle aspiration vs conventional transbronchial needle aspiration in the diagnosis of sarcoidosis. Chest 2014;146:547-56.

24. Dong X, Qiu X, Liu Q, et al. Endobronchial ultrasoundguided transbronchial needle aspiration in the mediastinal staging of non-small cell lung cancer: a meta-analysis. Ann Thorac Surg 2013;96:1502-7.

25. Chandra S, Nehra M, Agarwal D, et al. Diagnostic accuracy of endobronchial ultrasound-guided transbronchial needle biopsy in mediastinal lymphadenopathy: a systematic review and meta-analysis. Respir Care 2012;57:384-91.

26. Dhooria S, Aggarwal AN, Gupta D, et al. Utility and Safety of Endoscopic Ultrasound With BronchoscopeGuided Fine-Needle Aspiration in Mediastinal Lymph Node Sampling: Systematic Review and Meta-Analysis. Respir Care 2015;60:1040-50.

27. Hsia DW, Jensen KW, Curran-Everett D, et al. Diagnosis of lung nodules with peripheral/radial endobronchial ultrasound-guided transbronchial biopsy. J Bronchology Interv Pulmonol 2012;19:5-11.

28. Kurimoto N, Miyazawa T, Okimasa S, et al. Endobronchial ultrasonography using a guide sheath increases the ability to diagnose peripheral pulmonary lesions endoscopically. Chest 2004;126:959-65.

29. Yoshikawa M, Sukoh N, Yamazaki K, et al. Diagnostic value of endobronchial ultrasonography with a guide sheath for peripheral pulmonary lesions without X-ray fluoroscopy. Chest 2007;131:1788-93.

30. Kikuchi E, Yamazaki K, Sukoh N, et al. Endobronchial ultrasonography with guide-sheath for peripheral pulmonary lesions. Eur Respir J 2004;24:533-7.

31. Eberhardt R, Ernst A, Herth FJ. Ultrasound-guided transbronchial biopsy of solitary pulmonary nodules less than 20 mm. Eur Respir J 2009;34:1284-7.

32. Steinfort DP, Khor YH, Manser RL, et al. Radial probe endobronchial ultrasound for the diagnosis of peripheral lung cancer: systematic review and meta-analysis. EUR Respir J 2011;37:902-10.

33. Rivera MP, Mehta AC, Wahidi MM. Establishing the diagnosis of lung cancer: Diagnosis and management of lung cancer, 3rd ed: American College of Chest Physicians 
evidence-based clinical practice guidelines. Chest 2013;143:e142S-65S.

34. Eberhardt R, Anantham D, Herth F, et al. Electromagnetic navigation diagnostic bronchoscopy in peripheral lung lesions. Chest 2007;131:1800-5.

35. Makris D, Scherpereel A, Leroy S, et al. Electromagnetic navigation diagnostic bronchoscopy for small peripheral lung lesions. Eur Respir J 2007;29:1187-92.

36. Eberhardt R, Anantham D, Ernst A, et al. Multimodality bronchoscopic diagnosis of peripheral lung lesions: a randomized controlled trial. Am J Respir Crit Care Med 2007;176:36-41.

37. Gex G, Pralong JA, Combescure C, et al. Diagnostic yield and safety of electromagnetic navigation bronchoscopy for lung nodules: a systematic review and meta-analysis. Respiration 2014;87:165-76.

38. Zhang W, Chen S, Dong X, et al. Meta-analysis of the diagnostic yield and safety of electromagnetic navigation bronchoscopy for lung nodules. J Thorac Dis
2015;7:799-809.

39. Yabuuchi H, Murayama S, Sakai S, et al. Resected peripheral small cell carcinoma of the lung: computed tomographic-histologic correlation. J Thorac Imaging 1999;14:105-8.

40. Hashimoto M, Miyauchi T, Heianna J, et al. Accurate diagnosis of peripheral small cell lung cancer with computed tomography. Tohoku J Exp Med 2009;217:217-21.

41. Haque N, Raza A, McGoey R, et al. Small cell lung cancer: time to diagnosis and treatment. South Med J 2012;105:418-23.

42. Lee D, Rho JY, Kang S, et al. CT findings of small cell lung carcinoma: Can recognizable features be found? Medicine (Baltimore) 2016;95:e5426.

43. Brink I, Schumacher T, Mix M, et al. Impact of [18F] FDG-PET on the primary staging of small-cell lung cancer. Eur J Nucl Med Mol Imaging 2004;31:1614-20.
Cite this article as: Lai Y, Sun D, Bao P, Li W, Li X, Zhao J. An unexpected SCLC diagnosed by electromagnetic navigation bronchoscopy. J Thorac Dis 2018;10(11):E768-E773. doi: $10.21037 /$ jtd. 2018.10 .20 